Summary: Dispersing surface-modified zinc oxide nanoparticles $(\mathrm{ZnO})$ in methyl methacrylate (MMA) improves the free radical bulk polymerization process as well as the thermal stability of the formed polymer. Hydroxy groups available on the $\mathrm{ZnO}$ surface may induce a degenerative transfer. This suppresses the gel effect, which leads to a better control of the heat evolution during the late stages of polymerization. The formation of chains having vinylidene end groups and head-to-head links is suppressed, which shifts the onset of thermal decomposition to the regime where decomposition occurs by random chain scission.

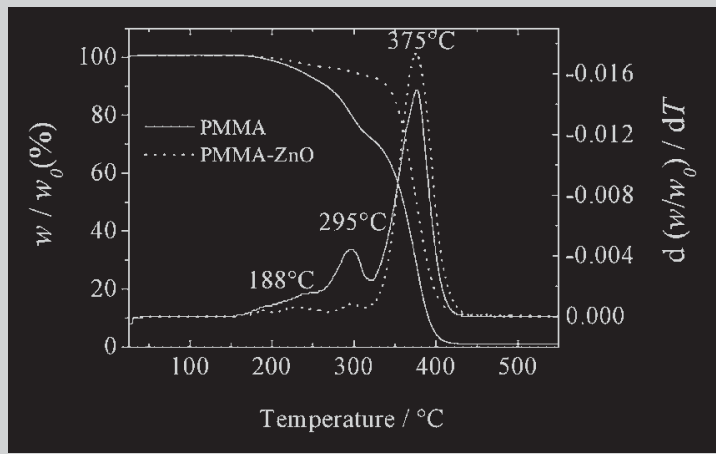

Thermal degradation profiles of PMMA and its composite with $\mathrm{ZnO}$ at $11 \mathrm{wt} . \%$.

\title{
PMMA/Zinc Oxide Nanocomposites Prepared by In-Situ Bulk Polymerization ${ }^{\mathrm{a}}$
}

\author{
Mustafa M. Demir, Mine Memesa, Patrice Castignolles, ${ }^{\mathrm{b}}$ Gerhard Wegner* \\ Max Planck Institute for Polymer Research, Ackermannweg 10, 55128 Mainz, Germany \\ Fax: +49 6131379 330; E-mail: wegner@mpip-mainz.mpg.de
}

Received: December 20, 2005; Revised: March 8, 2006; Accepted: March 9, 2006; DOI: 10.1002/marc.200500870

Keywords: composites; free radical polymerization; gel effect; nanoparticles; poly(methyl methacrylate); thermal degradation

\section{Introduction}

Dispersing nanometer-scale inorganic particles in a polymer matrix offers the potential of creating new materials, the properties of which arise from the synergies between the components. ${ }^{[1]}$ The crucial issue in preparing high quality composites is the uniform dispersion of the inorganics in the organic matrix. While it is notoriously difficult to achieve a homogeneous distribution of nanometer-scale particles in highly viscous polymer melts, and aggregation of the particles cannot be avoided, dispersion in a suitable monomer followed by polymerization in situ is an attractive alternative route. ${ }^{[2-4]}$ It is tacitly assumed that inorganic particles are not interfering with the mechanism of free radical polymerization; however, it will be shown in the

\footnotetext{
a $\square$ Supporting information for this article is available at the bottom of the article's abstract page, which can be accessed from the journal's homepage at http://www.mrc-journal.de, or from the author.

b Current address: Key Centre for Polymer Colloids, School of Chemistry F11, University of Sydney, NSW 2006, Australia.
}

following that this is not true by taking the bulk polymerization of methyl methacrylate (MMA) in the presence of nanometer-scaled $\mathrm{ZnO}$ as an example. In fact, it will be shown that degenerative chain transfer induced at the $\mathrm{ZnO}$ surface leads to a better control of chain growth, suppression of the gel effect, and improved thermal properties of bulk polymerized poly(methyl methacrylate) (PMMA). To the best of our knowledge, this is the first report of such kind demonstrating the potential semiconductor nanoparticles (here $\mathrm{ZnO}$ ) as controlling agents in free radical polymerization.

PMMA is an optically clear amorphous thermoplastic. It is widely used as a substitute for inorganic glass, because it shows higher impact strength and undergoes ductile rather than brittle fracture. It has favourable processing conditions, and a wide range of additives have been shown to further improve its properties. ${ }^{[5]} \mathrm{Zinc}$ oxide $(\mathrm{ZnO}$, 'zincite') is an environmentally friendly material. It is a colorless wide bandgap semiconductor with an optical bandgap in the UV region that makes it useful as an efficient absorber of UV radiation. It has a refractive index of 2.02 at 
$\lambda=589 \mathrm{~nm},{ }^{[6]}$ a thermal conductivity of $1.16 \mathrm{~W} \cdot \mathrm{m}^{-1} \cdot \mathrm{K}^{-1}$ at $50{ }^{\circ} \mathrm{C},{ }^{[7]}$ and an electrical conductivity of $0.02 \mathrm{~S} \cdot \mathrm{cm}^{-1},{ }^{[8]}$ all of these values being considerably higher than those of PMMA, $1.49,{ }^{[9]} 0.19 \mathrm{~W} \cdot \mathrm{m}^{-1} \cdot \mathrm{K}^{-1},{ }^{[9]}$ and $10^{-16} \mathrm{~S} \cdot \mathrm{cm}^{-1},[10]$ respectively, at the given conditions. The combination of these two materials should have many potential applications such as antireflection coatings, UV protecting films, transparent barrier/protective layers, and as flame-retardant materials. While composites of polyacrylate with $\mathrm{SiO}_{2}$ and $\mathrm{TiO}_{2}$ have found considerable attention, the polyacrylate/ $\mathrm{ZnO}$ pair has been rarely studied. Hung and Whang ${ }^{[1]}$ have reported on the luminescence of $\mathrm{ZnO} /$ poly (hydroxyethyl methacrylate) films where the $\mathrm{ZnO}$ particles had been produced by a sol-gel method and had been treated with a silane coupling agent before the polymerization was achieved in situ. Khrenov et al. ${ }^{[12]}$ produced surface-modified $\mathrm{ZnO}$ particles by employing a miniemulsion precipitation procedure and incorporated them into a PMMA matrix by blending. Liufu et al. ${ }^{[13]}$ investigated the thermal degradation of polyacrylate/ $\mathrm{ZnO}$ blends and proposed that the $\mathrm{ZnO}$ particles have both a role in stabilization and destabilization depending on the temperature region.

PMMA obtained by the free radical polymerization of MMA undergoes thermal degradation in three distinct steps. ${ }^{[14,15]}$ The first step at around $188^{\circ} \mathrm{C}$ and the second step at around $295^{\circ} \mathrm{C}$ are associated with degradation starting from head-to-head linkages and unsaturated chain ends followed by retropolymerization ('unzipping'). These two structural defects arise from the termination reaction in free radical polymerization by either combination or disproportionation. The major contribution to thermal decomposition arises from random chain scission which occurs with a maximum rate at around $375^{\circ} \mathrm{C}$. It will be shown that the presence of semiconductor particles like $\mathrm{ZnO}$ during polymerization has a profound effect on the thermal degradation. $\mathrm{ZnO}$ particles induce a chain stoppage reaction to take place at the filler surface. As a result of this chain stoppage, PMMA synthesized in the presence of $\mathrm{ZnO}$ contains less abnormal linkages, which inevitably form when the polymerization is done without filler because of conventional termination mechanisms. In this work, PMMA/ $\mathrm{ZnO}$ composites are prepared by bulk polymerization in the presence of nanosized semiconductor particles using 2,2'-azoisobutyronitrile (AIBN) as initiator. Effects of the inorganic particles on the polymerization, molecular weight distribution (MWD), and the thermal degradation of the composite material are investigated. The $\mathrm{ZnO}$ particles are of narrow size distribution centered at $25 \mathrm{~nm}$ with a cut-off at around $80 \mathrm{~nm}$ when dispersed into the monomer. The particles are hydrophobized by treatment with tert-butylphosphonic acid $\left(t \mathrm{BuPO}_{3} \mathrm{H}_{2}\right)$, which is necessary to prevent undesired aggregation and to increase the wettability by the monomer and polymer.

\section{Experimental Part}

\section{Materials}

Zinc acetate dihydrate $\left(\mathrm{Zn}(\mathrm{AcO})_{2} \cdot 2 \mathrm{H}_{2} \mathrm{O}\right.$, reagent ACS), pentan-1-ol (99\%), 2-methoxyethyl ether (99\%), and tertbutylphosphonic acid $\left(98 \%, t \mathrm{BuPO}_{3} \mathrm{H}_{2}\right)$ were provided by ACROS and used without purification. Tetrabutylammonium acetate (TBAc, $>90 \%$ ) was purchased from Fluka. $p$-Toluene sulfonic acid monohydrate $(99 \%)$ was obtained from Merck. Methyl methacrylate (MMA, Acros, stabilized with 1020 ppm hydroquinone mono methyl ether) was distilled under reduced pressure. AIBN (Fluka) was recrystallized from methanol.

\section{Particle Synthesis and Surface Modification}

$\mathrm{ZnO}$ nanoparticles were prepared via transesterification of zinc acetate dihydrate with pentan-1-ol at $130^{\circ} \mathrm{C}$. For a typical synthesis, $0.6 \mathrm{mmol}$ of $p$-toluene sulfonic acid monohydrate was dissolved in a mixture of 2-methoxyethyl ether $(8.5 \mathrm{~mL})$ and pentan-1-ol $(8.5 \mathrm{~mL}) . \mathrm{Zn}(\mathrm{AcO})_{2} \cdot 2 \mathrm{H}_{2} \mathrm{O}(2.3 \mathrm{mmol})$ was added to the solution. At $30 \mathrm{~min}$ of reaction time, i.e., after the desired $\mathrm{ZnO}$ nanosized crystals had formed, a mixture of $t \mathrm{BuPO}_{3} \mathrm{H}_{2}(0.8 \mathrm{mmol})$ and TBAc $(1.6 \mathrm{mmol})$ in a $3 \mathrm{~mL}$ equivolume mixture of pentan-1-ol and 2-methoxyethyl ether were added. The reaction was allowed to proceed for a further $30 \mathrm{~min}$. The particles protected at the surface by $t \mathrm{BuPO}_{3} \mathrm{H}_{2}$ were isolated by centrifugation at $4000 \mathrm{rpm}$ and washed with ethanol. Particle yield was $31 \pm 4 \%$.

\section{Polymerization}

Different amounts of the surface-modified $\mathrm{ZnO}$ nanoparticles and MMA ( $1 \mathrm{~mL}$ or $2.5 \mathrm{~mL}$ ) were placed into glass tubes sealed with septa. The weight fraction $(w)$ of $\mathrm{ZnO}$ was adjusted with respect to MMA. The dispersions were sonicated for $15 \mathrm{~min}$ and kept overnight in the dispersed state to achieve wetting of the particle surface. After a second sonication of $30 \mathrm{~min}$, AIBN (1.5 wt.- $\%$ ) was added and three cycles of a freeze-thaw process were applied prior to polymerization. The tubes were placed into a preheated bath at $60{ }^{\circ} \mathrm{C}$, the polymerization was performed under an argon atmosphere and it was stopped after the desired time by quenching to room temperature.

\section{Characterization}

${ }^{1} \mathrm{H}$ NMR spectra were obtained using a Bruker DPX 250 spectrometer. Samples were taken directly from the polymerization vessel without removing the particles from the mixture of monomer/polymer. Molecular weight distributions (MWDs) were determined by size exclusion chromatography (SEC) calibrated with PMMA standards using tetrahydrofuran (THF) as eluent at a flow rate of $1 \mathrm{~mL} \cdot \mathrm{min}^{-1}$. The polymers prepared without the presence of $\mathrm{ZnO}$ (reference samples) were directly analyzed by SEC after the residual monomer had been distilled off under vacuum. The samples obtained in the presence of $\mathrm{ZnO}$ were first diluted with acetone, and the nanoparticles were removed by centrifugation at $4000 \mathrm{rpm}$. The remaining polymer solution was poured into an excess of methanol whereupon the polymer precipitated. The precipitate 
was collected by centrifugation, and was then subjected to SEC analysis. Differential scanning calorimetry (DSC, Mettler Toledo TG5) and thermogravimetry (TGA, SDTA 851 Mettler Toledo) were carried out with a heating/cooling rate of $10 \mathrm{~K} \cdot \mathrm{min}^{-1}$ under nitrogen. The derivative thermogravimety (DTG) results were obtained by taking the temperature derivative of mass loss, $\mathrm{d}\left(w / w_{0}\right) / \mathrm{d} T$ at a constant heating rate where $w$ is the sample weight and $w_{0}$ is the initial sample weight. The size of the particles was determined by dynamic light scattering (DLS) using a Malvern Zetasizer 3000. X-Ray diffractograms were registered in a Seifert XRD 3000 TT diffractometer using a $\mathrm{Cu} \mathrm{K}_{\alpha}$ radiation source $(\lambda=$ $0.15418 \mathrm{~nm}$ ). Transmission electron microscopy (TEM) was carried out with Zeiss 912 Omega working at a voltage of $120 \mathrm{kV}$. Diffuse reflection infrared fourier transform (DRIFT) spectra were obtained from the powders using a Nicolet 730 spectrometer in the range of $4000-400 \mathrm{~cm}^{-1}$.

\section{Results and Discussion}

\section{Particle Synthesis and Characterization}

The X-ray diffraction pattern of the as-prepared $\mathrm{ZnO}$ powder has sharp reflections as expected for hexagonal crystalline $\mathrm{ZnO}$ (JCPDS card no: 36-1451). The surfactant $t \mathrm{BuPO}_{3} \mathrm{H}_{2}$ is added to the reaction medium in which the $\mathrm{ZnO}$ crystals are formed and is allowed to cover the particle surface in situ without isolating them. Vibrational spectroscopy confirms the interaction of $t \mathrm{BuPO}_{3} \mathrm{H}_{2}$ with the surface of the $\mathrm{ZnO}$ particles. DRIFT spectra of $t \mathrm{BuPO}_{3} \mathrm{H}_{2^{-}}$ modified $\mathrm{ZnO}$ particles are dominated by the vibrational bands of the metal-coordinated phosphonic acid molecules (see Supporting Information). The stretching bands of $\mathrm{P}-$ $\mathrm{OH}$ are observed at 1030 and $920 \mathrm{~cm}^{-1}$, together with a narrow band at $670 \mathrm{~cm}^{-1}$. The formation of such surface species has already been proposed by Cozzoli et al. for the surface modification of $\mathrm{ZnO}$ nanocrystals with $t \mathrm{Bu}$ $\mathrm{PO}_{3} \mathrm{H}_{2} \cdot{ }^{[16]}$ The broad $\mathrm{P}=\mathrm{O}$ stretching of $t \mathrm{BuPO}_{3} \mathrm{H}_{2}$ itself at $1200 \mathrm{~cm}^{-1}$ is not present because this band disappears when $t \mathrm{BuPO}_{3} \mathrm{H}_{2}$ is adsorbed to the surface of metal oxides. ${ }^{[17]}$ The absence of $\mathrm{P}=\mathrm{O}$ and presence of $\mathrm{P}-\mathrm{OH}$ stretching bands can be considered evidence for the successful anchoring of surfactant molecules to the $\mathrm{ZnO}$ surface via mainly bidentate and tridentate bonding modes. The graft density of the surfactant is calculated from the weight loss by thermogravimetry, and the surface area of $\mathrm{ZnO}$ particles is estimated from their known diameter. The graft density is found to be $2.5 \mu \mathrm{mol} \cdot \mathrm{m}^{-2}$ which corresponds to $50 \%$ coverage by phosphonic acid species. The area occupied by each surfactant molecule is equal to ca. $24 \AA^{2}$. 17$]$

\section{Polymerization: Conversion and MWD against Time}

The polymerization of MMA is conducted at $60{ }^{\circ} \mathrm{C}$ in bulk with $\mathrm{AIBN}$ as the initiator with the $\mathrm{ZnO}$ nanosized particles

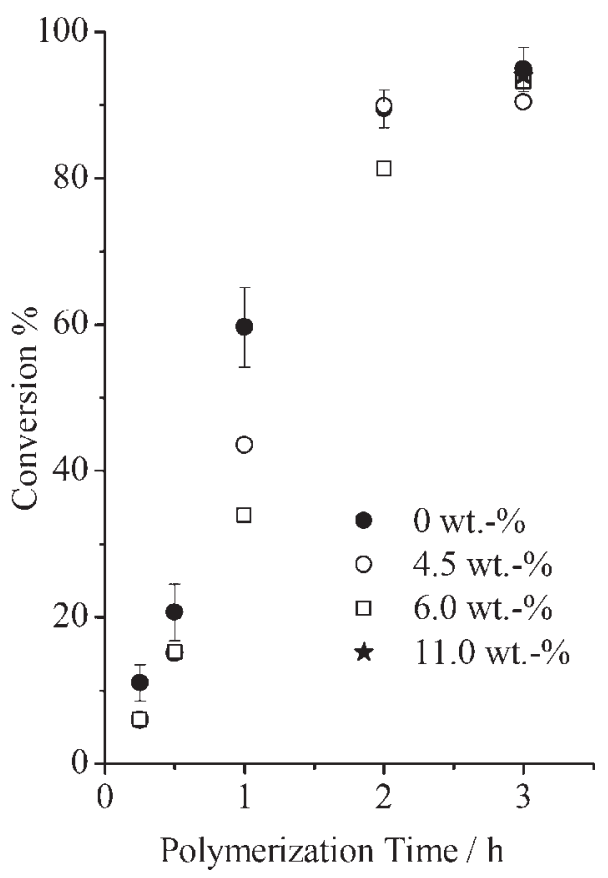

Figure 1. Conversions of MMA as a function of time in bulk polymerization at different weight fractions of $\mathrm{ZnO}$ nanoparticles at $60{ }^{\circ} \mathrm{C}, \mathrm{AIBN}(1.5 \mathrm{wt} .-\%)$ as initiator.

being present at weight fractions up to $11 \%$. The dispersion of the particles in the monomer is homogeneous, as indicated by the high translucency in the visible region.

Conversions are determined by ${ }^{1} \mathrm{H}$ NMR spectroscopy from the integration of the monomer signal (vinyl proton at $5.6 \mathrm{ppm})$ with respect to polymers $\left(\alpha-\mathrm{CH}_{3}\right.$ protons appear between 0.5 and $0.9 \mathrm{ppm}$ for different tacticities). ${ }^{[18]}$ Figure 1 shows conversion versus polymerization time for different weight fractions of particles; $0,4.5$, and $6.0 \mathrm{wt} .-\%$. In the very early stages of polymerization, the conversion vs. time curves have the same slope for all cases. However, the reference sample (MMA without particles) shows a distinct increase of slope between 20 and $60 \%$ conversion as a result of an autoacceleration phenomenon, even if the initiator, AIBN, has a half-time of only 16 min at $60{ }^{\circ} \mathrm{C} .{ }^{[19]}$ This noticeable increase in polymerization rate is known as the gel or Trommsdorff effect. Its origin is to be found in the increase of viscosity at even moderate polymer concentrations. ${ }^{[20]}$ Since termination is diffusion controlled, the rate of termination significantly decreases, and the free radical concentration increases, which causes a dramatic increase in the rate of polymerization. As the polymerization proceeds, a further increase of viscosity 'freezes' the reaction mixture and the rate coefficient of propagation drops. The polymerization slows down and levels off at $95 \%$ conversion after $3 \mathrm{~h}$. Contrary to this sigmoid conversion vs. time curve for the reference sample, the presence of the $\mathrm{ZnO}$ particles suppresses the Trommsdorff effect. However, a further classical indicator of the gel effect, namely strong 
broadening of the MWD on the high-molecular-weight side, ${ }^{[21]}$ is detected for all samples containing $\mathrm{ZnO}$ particles. In fact, without $\mathrm{ZnO}$, the MWD is systematically bimodal as shown in Figure 2a (see also Supporting
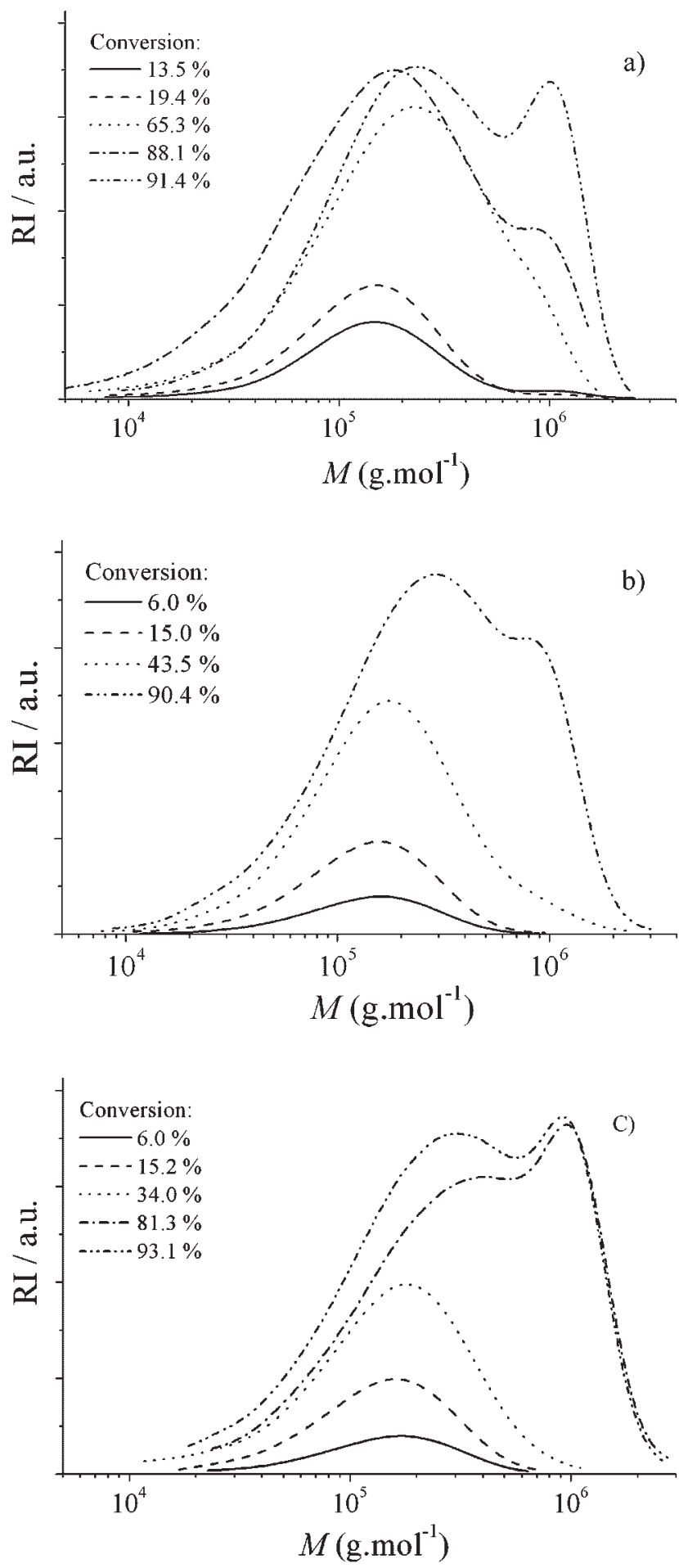

Figure 2. Molecular weight distributions normalized for conversion of three different weight fractions of $\mathrm{ZnO}$ nanoparticles: a) 0, b) 4.5 , and c) 6 wt.- $\%$.
Information). The greater the presence of $\mathrm{ZnO}$, the more the appearance of the very high molecular weight peak in the MWD is delayed (Figure $2 b$ and $c$ ).

\section{Size of the $\mathrm{ZnO}$ Particles in the Monomer and Polymer}

Diameter distributions of $\mathrm{ZnO}$ particles both in the monomer and as well as in the polymer (after the in situ bulk polymerization) are examined by DLS and TEM, respectively. The distribution of the particles in the polymer is obtained from TEM electromicrographs. Results of the measurements are presented in Figure 3 for two $\mathrm{ZnO}$ weight fractions, 6.0 and 11.0 wt.-\%. Surface-modified particles dispersed in the monomer exhibit a narrow size distribution with a mean diameter of $25 \mathrm{~nm}$ and a short tail extended to $80 \mathrm{~nm}$. DLS data provide the number size distribution of the primary particles. Consequently, they exhibit good dispersibility in the neat monomer without undergoing aggregation, even when the weight fraction is increased to $11 \mathrm{wt} .-\%$. On the contrary, after the polymerization, larger aggregates of primary $\mathrm{ZnO}$ particles are detected in the TEM images of the polymer/particle composites, Figure 4 . The aggregates, presented in the inset of Figure $4 \mathrm{a}$, consist of loosely associated individual particles $25 \mathrm{~nm}$ in diameter, which is consistent with the size measured by DLS. Clearly, polymerization induces a segregation of the primary particles to form domains within the bulk polymer, while increasing the particle content does not cause aggregation/segregation in the monomer as can be seen from the DLS distributions shown in Figure 3.

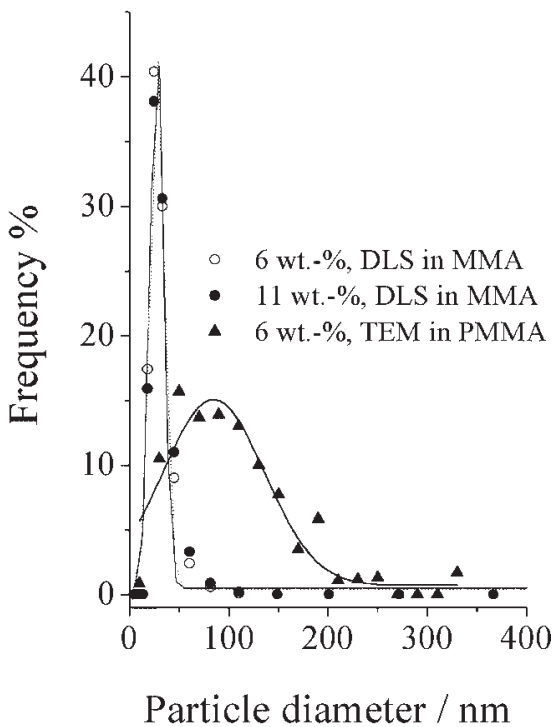

Figure 3. Diameter distributions of $\mathrm{ZnO}$ particles in MMA for 6 and 11 wt.- $\%$ and the distribution of aggregates in PMMA for 6 wt.-\%. 

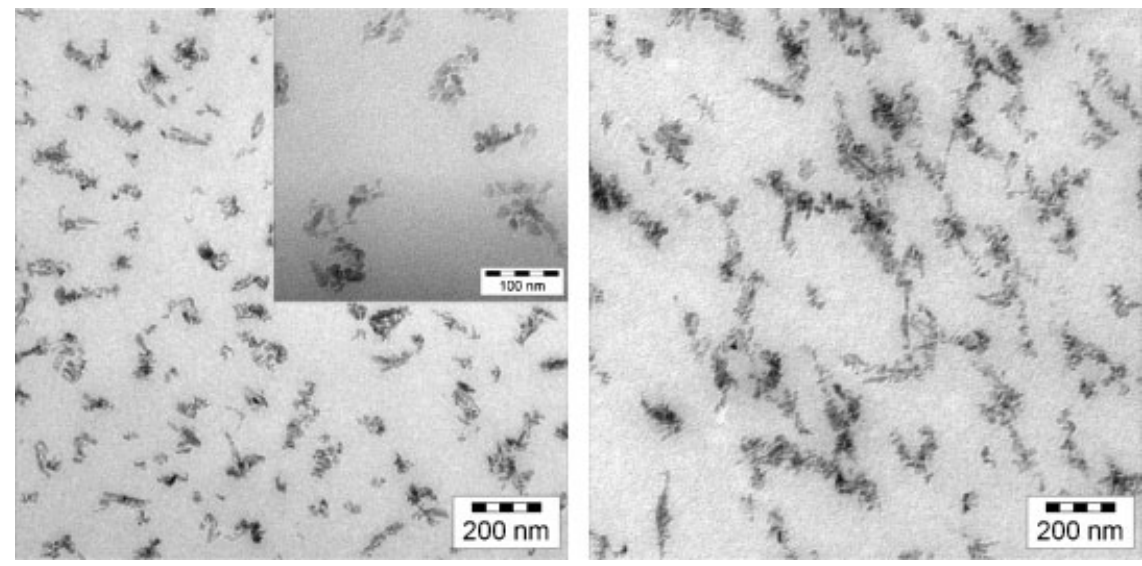

Figure 4. Bright field TEM images of thin sections of composites prepared by in-situ bulk polymerization with a) $6 \mathrm{wt} .-\%$ and b) $11 \mathrm{wt} .-\%$ of $\mathrm{ZnO}$ particles.

\section{Thermal Stability}

The thermal degradation profiles of PMMA and of a PMMA $/ \mathrm{ZnO}\left(w_{\mathrm{ZnO}}=11\right.$ wt. $\left.-\%\right)$ composite under nitrogen atmosphere are given in Figure 5a. The left and right axes refer to mass loss and the derivative of mass loss, respectively. The DTG of PMMA shows two minor steps at 188 and $295^{\circ} \mathrm{C}$ and a major one at $375^{\circ} \mathrm{C}$. The thermogram agrees with the earlier published data. ${ }^{[14,15]}$ The first two peaks are attributed to thermal degradation initiated from the head-to-head linkages arising from chain termination by combination and vinylidene chain ends that result from disproportionation. The mass loss associated with the second peak is higher than the one connected to the first peak because, at $60{ }^{\circ} \mathrm{C}, 79 \%$ of the terminations in the MMA free radical polymerization occur by disproportionation. ${ }^{[22]}$ The major peak refers to degradation initiated by random scission of the polymer backbone. In contrast to PMMA prepared in the absence of $\mathrm{ZnO}$, the DTG curve of the PMMA/ZnO composite shows only one sharp peak at $375^{\circ} \mathrm{C}$, which is indicative of degradation resulting from the random scission of the backbone. Peaks that originate from the degradation started at the head-to-head linkages and vinylidene chain ends have practically disappeared, and the onset of degradation is retarded from 170 to $340{ }^{\circ} \mathrm{C}$ in the polymer prepared in the presence of $\mathrm{ZnO}$. The disappearance of these peaks occurs gradually with increasing particle weight fraction but is very significant even at only $4.5 \mathrm{wt}$.$\%$ of $\mathrm{ZnO}$ (see Supporting Information). The weight loss for samples subject to heating to a temperature of $318^{\circ} \mathrm{C}$ is $27 \%$ for PMMA prepared in absence of $\mathrm{ZnO}$, whereas for the composites having 4.5 and $11 \mathrm{wt} .-\%$ of $\mathrm{ZnO}$, the losses are 8.5 and $6.3 \%$, respectively. The strong reduction of the peak at $295^{\circ} \mathrm{C}$ in the DTG of the composites shows that the formation of vinylidene end groups is significantly suppressed when the polymerization proceeds in the presence of $\mathrm{ZnO}$ nanosized particles.

\section{Mechanism of the Polymerization}

The results prove that in the presence of $\mathrm{ZnO}$ the rate of polymerization, the molecular weight distribution, and the microstructure of the polymer are changed. These effects are consistent with the literature dealing with free radical photopolymerization of MMA in the presence of $\mathrm{ZnO}$ colloids. ${ }^{[23]}$ Bourgeat-Lami ${ }^{[24]}$ reviewed the effect of inorganics, for example clay and $\mathrm{CaSO}_{3}$, on the kinetics of multi-phase polymerization of vinyl monomers. The author indicated that the studies reported so far are highly controversial and pointed out that the nature of the particle surface is the key parameter to understanding the in-situ polymerization.

Taking into account the reactivity of a free radical and the presence of nanometer-scale semiconductor particles, an oxidation-reduction reaction between the radical and the particles might be expected. In fact, the transfer of electrons from radicals to quantum sized (Q) $\mathrm{ZnO}$ nanoparticles was shown by Haase et al. ${ }^{[25]}$ Electrons were injected from radiolytically generated $\mathrm{CH}_{2} \mathrm{OH}$ radicals and accumulated at the particle surface upon UV illumination. Later, upon photopolymerizing MMA using the same $\mathrm{Q}$-sized $\mathrm{ZnO}$ particles as photoinitiator, Hoffman et al. ${ }^{[23]}$ suggested that the electrons situated on the particle surface initiate the polymerization via electron transfer in the first step, while the propagation eventually takes place by a free radical mechanism. In the present situation, i.e., the polymerization of a mixture of MMA and $\mathrm{ZnO}$ without illumination and with particles larger than Q-sized, a direct reaction between MMA and $\mathrm{ZnO}$ does not take place but rather AIBN is necessary to initiate the reaction. However, in our case, $\mathrm{ZnO}$ reacts with the propagating free radicals. First, the initiating radicals might be trapped at the surface to decrease the initiator efficiency as well as the free radical concentration. This hypothesis is not supported by experimental evidence, since the rate of polymerization and the MWDs 

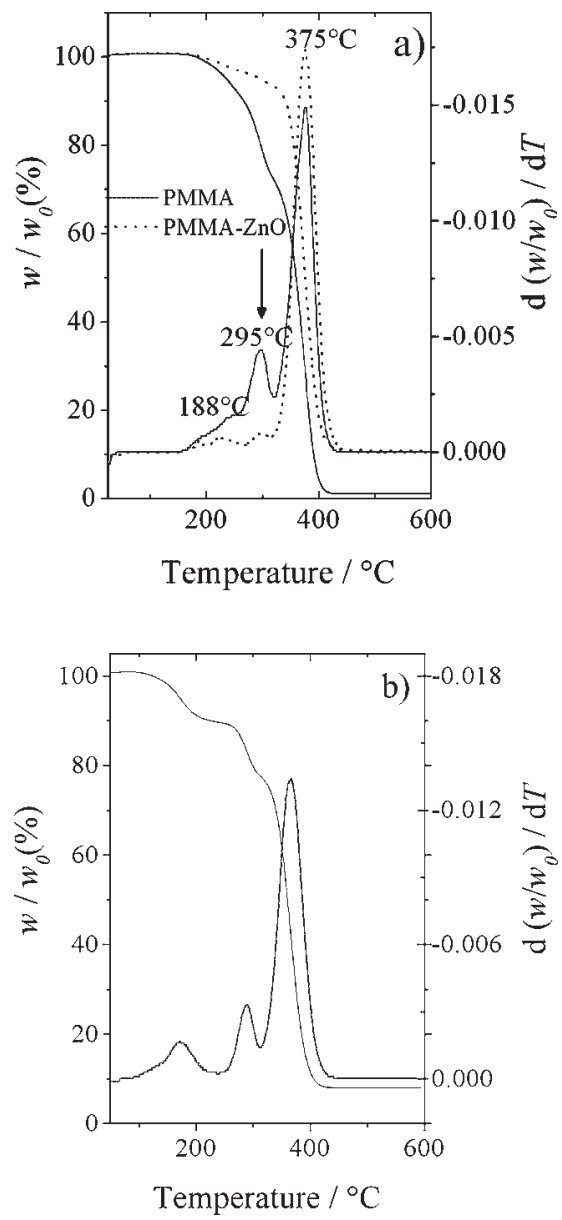

Figure 5. Thermal decomposition of a) (-) PMMA and ( $\cdots$. a a PMMA/ZnO composite $\left(w_{\mathrm{ZnO}}=11\right.$ wt.- $\left.\%\right)$ prepared by bulk polymerization over $3 \mathrm{~h}$ under nitrogen flow, b) a blend of preformed PMMA and $\mathrm{ZnO}\left(w_{\mathrm{ZnO}}=8.0\right.$ wt. $\left.-\%\right)$.

are identical at low conversion with or without $\mathrm{ZnO}$. Second, $\mathrm{ZnO}$ could terminate some free radicals either by disproportionation or by combination. The combination mechanism should lead to chemical grafting of PMMA onto $\mathrm{ZnO}$. The size of the $\mathrm{ZnO}$ particles before and after the polymerization (after washing the particles with acetone to remove physisorbed chains on the particle surface) is the same (ca. $25 \mathrm{~nm}$ ), which proves that grafting does not take place. The disproportionation mechanism implies the formation of a vinylidene end group as a consequence of $\beta$-hydrogen abstraction from the terminal radical by reaction with the $\mathrm{ZnO}$ surface. The presence of vinylidene end groups is disproved by the TGA results. Third, it is to be assumed that the surface of metal oxides is covered with a layer of hydroxy groups ${ }^{[26]}$ which may be reactive in terms of transfer reactions. If the $\mathrm{ZnO}$ behaves as a classical transfer agent, the MWD will shift towards lower molecular weights. However, this is not observed at all. In fact, if an $\mathrm{H}$ atom is abstracted from the hydroxy groups presented by the $\mathrm{ZnO}$ surface, it is unlikely that the radicals formed at the $\mathrm{ZnO}$ surface would reinitiate instantaneously, which is the condition required for a classical chain transfer. Thus, it seems that a degenerative transfer takes place. An $\mathrm{H}$ atom is transferred from the $-\mathrm{OH}$ group on the $\mathrm{ZnO}$ surface to the propagating radical. The surface-bound radical cannot directly initiate the growth of new polymer chains nor can it combine with polymeric free radicals for this would lead to grafting, which is not observed. However, the TGA proves that the conventional termination events are greatly disfavored, while the rate of polymerization remains of the order of magnitude of the control experiment (without $\mathrm{ZnO}$ ). If the radicals created on the $\mathrm{ZnO}$ surface are too stable to further react and play a role in the polymerization, $\mathrm{ZnO}$ would act as an inhibitor and inhibition would lower the rate of polymerization within one polymerization. The rate of polymerization is observed to be constant within one experiment in the presence of $\mathrm{ZnO}$, thus discarding the possibility of inhibition. There are two consequences: i) most of the chain stoppage may occur by the degenerative chain transfer to the $\mathrm{ZnO}$ surface, ii) the radicals created on the $\mathrm{ZnO}$ surface may induce a slow reinitiation, not directly reacting with the monomer, but with other species present, for example, the water physiosorbed on the $\mathrm{ZnO}$ surface (the presence of which is proven by vibrational spectroscopy). In this case, hydroxyl radicals could be released from the surface and play the role of initiators.

The mechanism of the polymerization may thus contain two competitive chain stoppage events: conventional termination and degenerative transfer to $\mathrm{ZnO}$. At low conversion, the rate of termination is faster and the influence of the presence of $\mathrm{ZnO}$ is very weak. At intermediate conversion, the increase in viscosity leads to a significant decrease of the coefficient of termination, and so the degenerative transfer to $\mathrm{ZnO}$ becomes faster than termination and becomes the predominant chain stoppage event.

The autoacceleration that is macroscopically observed is related to very strong local heat evolution which produces a second population in the high-molecular-weight region of the MWD. The presence of $\mathrm{ZnO}$ limits both the macroscopic autoacceleration and the local heat evolution (compare the high-molecular-weight peaks of Figure 2). It may be worth mentioning that the addition of neat $t \mathrm{BuPO}_{3} \mathrm{H}_{2}$ to the polymerization medium had no effect of all.

\section{Properties of the Materials}

Rather than nanocomposite preparation via in-situ polymerization, blending of the same $\mathrm{ZnO}$ particles $\left(w_{\mathrm{ZnO}}=\right.$ 8 wt.-\%) with preformed PMMA is also examined. The 
degradation path of this composite is shown in Figure $5 b$ and is very similar to that of pure PMMA. The $\mathrm{ZnO}$ particles do not have a significant effect on the thermal stability of the composite prepared by blending. However, the composites prepared by in-situ polymerization exhibit superior thermal stability compared to that obtained from a simple blending. In other words, the preparation method has a direct influence on the thermal degradation of the composite materials. The glass transition temperature $\left(T_{\mathrm{g}}\right)$ of nanocomposites measured by DSC (see Supporting Information) is found to be positioned between 102 to $109^{\circ} \mathrm{C}$, very close to the $T_{\mathrm{g}}$ of atactic PMMA $\left(105^{\circ} \mathrm{C}\right)$. In other words, the presence of nanosized particles does not seem to have an influence on the glass transition, at least not in the range of loading up to $11 \mathrm{wt} .-\%$. This is further evidence that there is no specific interaction between the particles and the polymer matrix. As for transparency of the PMMA/ZnO composites prepared by bulk polymerization, a detailed characterization is under way. At this time, a distinct cut-off of transmission at $370 \mathrm{~nm}$ towards a shorter wavelength as a result of the high absorption coefficient of $\mathrm{ZnO}$ has been seen. Transmission data of the nanocomposite films at $550 \mathrm{~nm}$, at which the human eye has maximum sensitivity, ${ }^{[27]}$ and at $350 \mathrm{~nm}$ are examined. While films of $2.0 \mu \mathrm{m}$ thickness do not display a significant transmission loss in the visible region ( $91 \%$ on average), a strong filter effect is observed in the $\mathrm{UV}$ region as the $\mathrm{ZnO}$ content increases with an initial slope of -4.8 loss in transmission per weight percentage. At the employed film thickness and $\mathrm{ZnO}$ content, and since the scattering that results from large $\mathrm{ZnO}$ aggregates is avoided, the composite films are transparent and have strong UV-protection capability. Even thick samples of up to $1 \mathrm{~mm}$ thickness are highly translucent between the wavelengths of 400 and $800 \mathrm{~nm}$.

\section{Conclusion}

The bulk polymerization of MMA in the presence of up to 11 wt.-\% of nanosized $\mathrm{ZnO}$ (zincite) particles gives access to composite materials, which show an improved resistance to thermal degradation in comparison to blends of PMMA with the same $\mathrm{ZnO}$ particles. The presence of the $\mathrm{ZnO}$ particles in bulk polymerization suppresses the Trommsdorff effect and leads to a better-defined MWD at high conversions.

The origin of these effects is seen in the reactivity of the $\mathrm{ZnO}$ surface towards the propagating chain-end radicals of PMMA. A hypothesis is put forward that hydroxy groups, inevitably present at the $\mathrm{ZnO}$ particle surface, may act as the sites at which degenerative transfer by hydrogen abstraction may take place and that chain termination by combination and/or disproportionation is thereby competitively suppressed. A slow release of radical species that may also involve surface-bound water or similar species from the $\mathrm{ZnO}$ surface may take place and contribute to the overall rate of reaction as well as to the observed MWD. It is important to note that surface grafting to the $\mathrm{ZnO}$ particles does not take place and that the particles can be retrieved from the blend unchanged by dissolving away the polymer. Thus, there is no specific physical interaction between the particles and the polymer in the bulk of the blend. This may be a consequence of the modification of the particle surface by tert-butylphosphonic acid leading to a ca. 50\% monolayer coverage but leaving a sufficient number of free hydroxy groups available for interaction with the growing chain radicals. The surface modification by tert-butylphosphonic acid is necessary to achieve wetting of the particles by the monomer and polymer and to prevent undesired aggregation.

[1] G. Kickelbick, Prog. Polym. Sci. 2003, 28, 83.

[2] J. Jordan, K. I. Jacob, R. Tannenbaum, M. A. Sharaf, I. Jasiuk, Mater. Sci. Eng. A 2005, 393, 1.

[3] T. Kashiwagi, A. B. Morgan, J. M. Antonucci, M. R. VanLandingham, R. H. Harris, W. H. Awad, J. R. Shields, J. Appl. Polym. Sci. 2003, 89, 2072.

[4] M. Avella, M. E. Errico, E. Martuscelli, Nano Lett. 2001, 1, 213.

[5] B. B. Kine, R. W. Novak, "Acrylic and Methacrylic Ester Polymers", in: Encyclopedia of Polymer Science and Engineering, $2^{\text {nd }}$ edition, H. F. Mark, N. M. Bikales, C. G. Overberger, G. Menges, J. I. Kroschwitz, Eds., Wiley, New York 1985, Vol. 1, p. 262.

[6] D. R. Lide, Ed., "CRC Handbook of Chemistry and Physics", $76^{\text {th }}$ edition, CRC Press, Boca Raton, FL 1995, p. $4 / 138$

[7] T. Olorunyolemi, A. Birnboim, Y. Carmel, O. C. Wilson, I. K. Lloyd, S. Smith, R. Campbell, J. Am. Ceram. Soc. 2002, $85,1249$.

[8] D. C. Look, Semicond. Sci. Technol. 2005, 20, S55.

[9] W. Wunderlich, "Physical Constants of Poly(methyl methacrylate)", in: Polymer Handbook, $4^{\text {th }}$ edition, J. Brandrup, E. H. Immergut, E. A. Grulke, Eds., J. Wiley \& Sons, New York, USA 1999, p. V/87.

[10] W. G. Zheng, S. C. Wong, H. J. Sue, Polymer 2002, 43, 6767.

[11] C. H. Hung, W. T. Whang, J. Mater. Chem. 2005, 15, 267.

[12] V. Khrenov, M. Klapper, M. Koch, K. Müllen, Macromol. Chem. Phys. 2005, 206, 95.

[13] S. C. Liufu, H. N. Xiao, Y. P. Li, Polym. Degrad. Stab. 2005, 87, 103.

[14] L. E. Manring, D. Y. Sogah, G. M. Cohen, Macromolecules 1989, 22, 4652

[15] T. Kashiwagi, A. Inaba, J. E. Brown, K. Hatada, T. Kitayama, E. Masuda, Macromolecules 1986, 19, 2160.

[16] P. Davide Cozzoli, M. Lucia Curri, A. Agostiano, G. Leo, M. Lomascolo, J. Phys. Chem. B 2003, 107, 4756.

[17] [17a] G. Guerrero, P. H. Mutin, A. Vioux, Chem. Mater. 2001, 13, 4367; [17b] J. Randon, P. Blanc, R. Paterson, J. Membr. Sci. 1995, 98, 119.

[18] J. Rudolph, J. Patzsch, W. H. Meyer, G. Wegner, Acta Polym. 1993, 44, 230. 
[19] K. W. Dixon, "Decomposition Rates of Organic Free Radical Initiators", in: Polymer Handbook, $4^{\text {th }}$ edition, J. Brandrup, E. H. Immergut, E. A. Grulke, Eds., J. Wiley \& Sons, New York, USA 1999, p. II/1.

[20] [20a] E. Trommsdorff, H. Köhle, P. Lagally, Macromol. Chem. 1948, 1, 169; [20b] R. G. V. Norrish, R. R. Smith, Nature 1942, 150, 336; [20c] M. Buback, M. Egorov, R. G. Gilbert, V. Kaminsky, O. F. Olaj, G. T. Russell, P. Vana, G. Zifferer, Macromol. Chem. Phys. 2002, 203, 2570 .

[21] G. A. Oneil, M. B. Wisnudel, J. M. Torkelson, Macromolecules 1996, 29, 7477.

[22] H. R. Allock, F. W. Lampe, "Contemporary Polymer Chemistry", $2^{\text {nd }}$ edition, Prentice Hall, Englewood Cliffs, NJ 1981, p. 61.
[23] A. J. Hoffman, H. Yee, G. Mills, M. R. Hoffmann, J. Phys. Chem. 1992, 96, 5540.

[24] [24a] E. Bourgeat-Lami, "Organic-Inorganic Nanocomposites by Multiphase Polymerization", in: Dendrimers, Assemblies and Nanocomposites, R. Arshady, A. Guyot, Eds., Citus Book, London 2002, Vol. 5, Ch. 5, pp. 149194; [24b] J. H. Yeum, Y. L. Deng, Colloid Polym. Sci. 2005, $283,1172$.

[25] M. Haase, H. Weller, A. Henglein, J. Phys. Chem. 1988, 92 , 482.

[26] E. McCafferty, J. P. Wightman, Surf. Interface Anal. 1998, $26,549$.

[27] E. M. C. Fortunato, P. M. C. Barquinha, A. C. M. B. G. Pimentel, A. M. F. Goncalves, A. J. S. Marques, L. M. N. Pereia, R. F. P. Martins, Adv. Mater. 2005, 17, 590. 